\title{
Optical switches using electro-optical effects in liquid crystals
}

\author{
Yu. Semenova ${ }^{* a}$, S.M. Dovgalets**b ${ }^{*}$ Yu. Panarin $*^{* a}$, G. Farrel1*a \\ ${ }^{a}$ School of Electronic \& Communications Engineering, Dublin Institute of Technology, \\ Kevin Street, Dublin 8, Ireland \\ ${ }^{b}$ Department of Automatics \& Information-Measuring Engineering, Vinnitsa State Technical \\ University, Khmelnitskoe Shosse 95, 21021 Vinnitsa, Ukraine \\ Copyright 2002 Society of Photo-Optical Instrumentation Engineers.
}

This paper will be published in SPIE Proceedings of Opto-Ireland Conference, Galway 2002 and is made available as an electronic preprint with permission of SPIE. One print or electronic copy may be made for personal use only. Systematic or multiple reproduction, distribution to multiple locations via electronic or other means, duplication of any material in this paper for a fee or for commercial purposes, or modification of the content of the paper are prohibited.

\begin{abstract}
Optical switches using two electro-optical effects in liquid crystals are proposed and investigated for incorporation in a switch matrix for optical networks. These two devices employ selective reflection in cholesteric layers and total reflection at the border between glass and nematic liquid crystal. Prototypes of these switches have been designed and their parameters have been investigated. Initial results show the switching contrast ratio of 38,5 and $42 \mathrm{~dB}$, respectively, with insertion loss about 2.3...2.9 dB for polarized light.
\end{abstract}

Keywords: All-optical matrix switch, liquid crystal, cholesteric mirror, total reflection

\section{INTRODUCTION}

All-optical switching fabrics will be a significant component in order to relieve the capacity limitation of electronicswitched networks. These devices allow to switch the traffic directly in the optical domain, avoiding the need of several optical $\leftrightarrow$ electrical conversions.

Several technologies have been proposed as candidates for optical switching. The most common switching technology is based on optomechanical switches, where the path of light is switched by some form of mechanical movement of either fibers or mirrors. Such switches feature good scalability, however, this approach involves moving parts, and typically has a limited lifetime of up to $10^{6}$ cycles. Some of the best developed types of optical switch employ liquid crystal (LC) materials due to their extreme sensitivity to applied fields, low power consumption, long lifetime and to their low cost.

Several different physical mechanisms for LC switches have been investigated and tested. One LC technology involves polymers containing nematic LC droplets [1]. Another approach involves chiral smectic A, which has a much faster response (10 $\mathrm{s}$ s versus a few ms) [2]. Some ferroelectric liquid crystal switching technologies are based on the concept of total internal reflection [3,4]. However, these approaches are characterized by a high energy loss and thus high insertion loss, which limits the practical applications of LC optical switches in optical networks.

The most popular form of switching technology is a so-called matrix switch. We have considered the matrix consisting of $2 \times 2$ single switches as a basic structure. This structure can be subsequently incorporated into a module containing $\mathrm{N}^{2}$ basic matrices (Fig.1) 


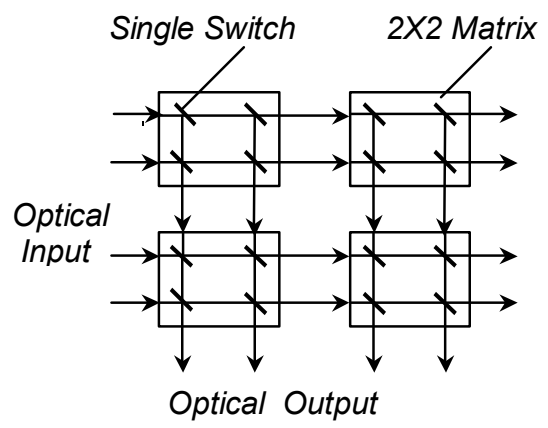

Figure1. Matrix spatial switch.

In this paper we present the design and comparison of two possible all-optical switching devices based on electro-optical effects in liquid crystals:

- Passive cholesteric mirror

- Total reflection switch

Experimental results on these designs investigations will be presented.

\section{PRINCIPLES OF OPERATION}

The first LC device demonstrated used a cholesteric mirror and electrically controllable nematic LC wave plate to achieve switching (Fig.2). The nematic LC wave plate functions as an electronically controlled phase retarder. As it is known, an input beam that is normally incident on the wave plate will be resolved into ordinary and extraordinary axis components, each with a different refractive index. The beam that emerges has a phase-delay difference or retardation between the axes

$$
\Gamma=\frac{2 \pi\left(n_{e}-n_{o}\right) d}{\lambda},
$$

where $\lambda$ is radiation wavelength, $n_{e}, n_{o}$ - the liquid crystal material refractive indices and $d$ is the material thickness.

A phase shift $\Gamma=\pi / 2$ will convert linearly polarized light to right circular polarized in case of the plate's optic axis oriented by $45^{\circ}$ to the direction of input light polarization, whereas a retardation $\Gamma=3 \pi / 2$ will result in left circular polarized light under the same conditions.
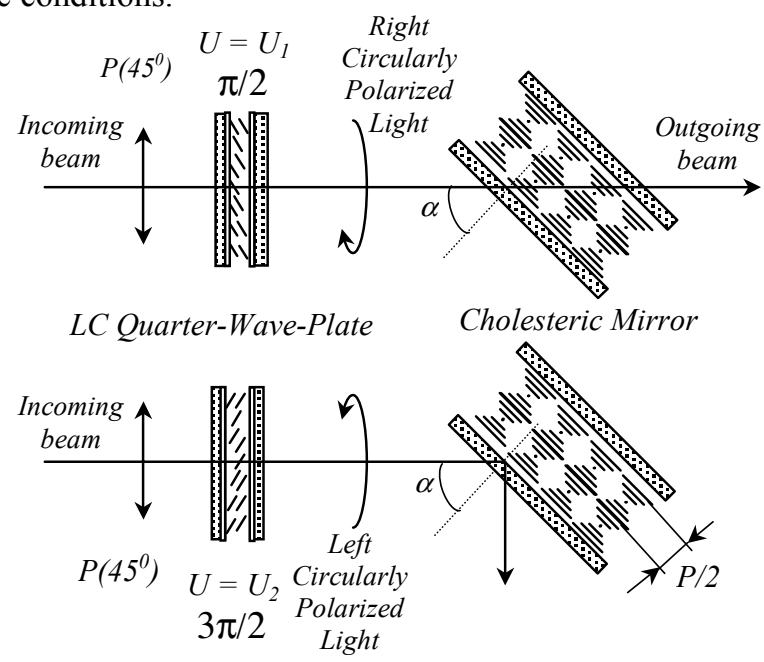

Figure 2.Cholesteric mirror switch schematic diagram. 
A standard nematic liquid crystal cell with planar surface conditions provides tunable phase retardation by changing the effective birefringence of the material with applied voltage. The cell can be adjusted to achieve the retardation values of $\pi / 2$ and $3 \pi / 2$ at a certain wavelength in the range from visible to IR region

A cholesteric mirror is used as a key element of the switch. Cholesteric LC layers have the ability to reflect right- or left-circularly polarized light of the handedness and wavelength that matches the twist sense and the helical pitch (multiplied by the refractive index of the material) in the cholesteric layer:

$$
\lambda_{0}=n_{a v} p \cos \alpha \text { with } n_{a v}^{2}=\frac{n_{e}^{2}+n_{0}^{2}}{2},
$$

where $p$ is cholesteric helical pitch, $\alpha$ is the angle of the input beam incidence. Light at other wavelengths or other polarization states is transmitted. The width of the reflection band $\Delta \lambda$ equals

$$
\Delta \lambda=\lambda_{0} \frac{\Delta n}{n_{a v}} .
$$

Thus, a cholesteric cell can transmit or reflect the incident light depending on the polarization state if the LC material parameters $\left(p, n_{a v}\right)$ and the incident angle $(\alpha)$ satisfy the selective reflection condition (2). So, it is possible to switch the path of the input light by changing of the light polarization, that is can be achieved by applying of an appropriate voltage value to the controllable LC wave plate.

Schematic diagram of the total internal reflection switch is shown in the Figure 3. Planar nematic LC layer is sandwiched between two glass prisms with transparent conducting and aligning layers.

The glass and LC material's refractive indices should be adjusted to maintain the switch transparency in the OFF-state and total reflection condition in the ON-state. The alignment layers should provide a homogeneous planar orientation with the director parallel to the incident light polarization direction. The required thickness of LC layer should be much greater than the decay distance for the evanescent wave. Without an applied voltage the nematic has a refractive index $\left(n_{L C-O F F}\right)$ near that of the glass prisms $\left(n_{g}\right)$, so the incident light passes through the liquid crystal layer. Liquid crystal molecules change their orientation while the switch is turned on by the applied voltage, so the LC refractive index becomes smaller than that of the glass $\left(n_{L C-O N}<n_{g}\right)$ to satisfy the total internal reflection condition, so the input beam will be reflected.

Let consider a monochromatic plane wave propagating in an arbitrary direction through nematic liquid crystal; two independent, linearly polarized, propagation modes can exist whose phase velocities are determined by the indices of refraction $n_{A}$ and $n_{B}$ along the each direction of polarization. The intersection of the material's index ellipsoid

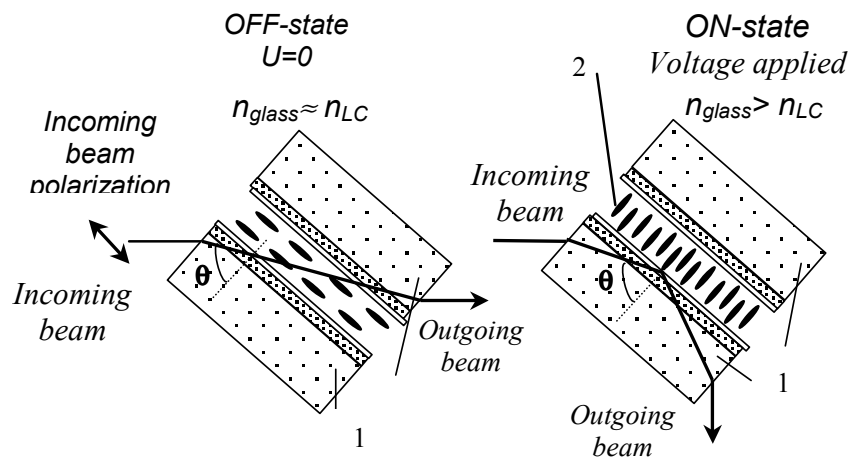

Figure 3. Total reflection switch structure: 1- glass prisms; 2- liquid crystal layer.

$$
\frac{x^{2}}{n_{x}{ }^{2}}+\frac{y^{2}}{n_{y}{ }^{2}}+\frac{z^{2}}{n_{z}{ }^{2}}=1,
$$

and the plane normal to the direction of propagation produce an ellipse whose major and minor semi-axes determine the indices $n_{A}$ and $n_{B}$. For nematic liquid crystal these indices depend on the surface alignment conditions (or the texture type) and on the strength and direction of the electric field applied to the electrodes. Without an applied electric field the 
nematic director is parallel to the electrodes surfaces and is modeled as a uniaxial crystal with a principal axis ellipsoid given by:

$$
\frac{x^{2}}{n_{e}{ }^{2}}+\frac{y^{2}}{n_{o}{ }^{2}}+\frac{z^{2}}{n_{o}{ }^{2}}=1 .
$$

The intersection of the normal plane for an incident linearly polarized plane wave has indices of refraction given by:

$$
\begin{gathered}
n_{A}=n_{z}=n_{o}, \\
n_{B}=\left\{\frac{\cos ^{2} \theta}{n_{e}^{2}}+\frac{\sin ^{2} \theta}{n_{o}^{2}}\right\}^{-1 / 2},
\end{gathered}
$$

where $n_{o}, n_{e}$ - liquid crystal refracting indices, $\theta$ - angle between the light propagation direction and the crystal surface.

Thus, for the light with its $\mathbf{E}$ component lying in the plane formed by the $\mathbf{k}$ vector of the beam and the crystal optical axis, the refractive index is $n_{L C-O F F}=n_{B}$.

Under an applied electric field liquid crystal molecules change their orientation along the field, so the nematic layer becomes homeotropically aligned and the crystal axis is along the y-axis.

For a linearly polarized beam propagating through the cell in the ON-state the index of refraction is given by:

$$
n_{B}=\left\{\frac{\sin ^{2} \theta}{n_{e}^{2}}+\frac{\cos ^{2} \theta}{n_{o}^{2}}\right\}^{-1 / 2}
$$

for the case when $\mathbf{E}$-component is lying in the plane formed by the $\mathbf{k}$-vector and the LC optical axis.

In this way the LC optical axis changes its orientation depending on applied electric field value. It gives a possibility to vary the LC refractive index in the range from $n_{L C-O F F}$ to $n_{L C-O N}$. As can be seen from Fig.4, the difference between $n_{L C-O F F}$ and $n_{L C-O N}$ values depends on both the LC optical anisotropy and the angle of input beam incidence.

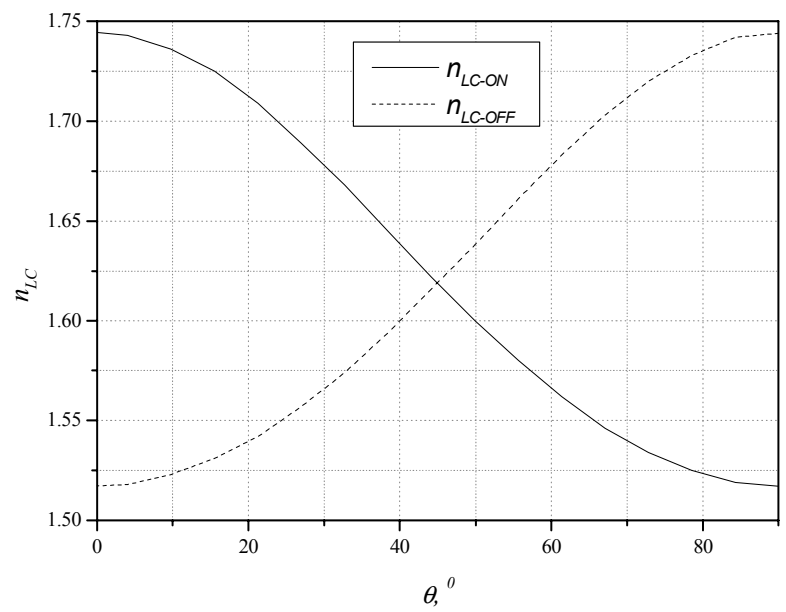

Figure 4. Calculated dependencies of refracting indices on the angle of incidence for OFF and ON modes. 
A planar liquid crystal layer may be considered as a conventional Fabri-Perot interferometer with a refractive index $n_{L C}$ depending on applied voltage which light transmittance is given by the formula:

$$
I=\frac{I_{0}}{1+M \cdot \sin ^{2}(\phi)}
$$

where $I$ is the intensity of the light transmitted by liquid crystal layer; $I_{0}$ is the intensity of the incident light;

$$
\phi=k \cdot n_{L C} \cdot 2 d \cdot \cos \left(\theta_{R}\right)
$$

is the phase difference between two beams reflected by the layer; $d$ is the liquid crystal layer thickness; $k=\frac{2 \pi}{\lambda}$ is the wave number in vacuum; $\lambda$ - radiation wavelength; $\theta_{R}$ - angle of refraction at the glass-LC interface which is given by Snell's law:

$$
n_{\text {glass }} \cdot \sin (\theta)=n_{L C} \cdot \sin \left(\theta_{R}\right),
$$

where $\theta$ is the angle of incidence;

$$
M=\frac{4 R}{1-R^{2}},
$$

and $R=\frac{I_{r}}{I_{o}}$ - intensity of the reflected light $I_{r}$ at the glass-liquid crystal interface related to the incident intensity $I_{0}$ which is given by:

$$
R_{\perp}=\frac{\sin ^{2}\left(\theta_{R}-\theta\right)}{\sin ^{2}\left(\theta_{R}+\theta\right)}
$$

for the light with its $\mathbf{E}$ component perpendicular to the plane of incidence and

$$
R_{\|}=\frac{\tan ^{2}\left(\theta-\theta_{R}\right)}{\tan ^{2}\left(\theta+\theta_{R}\right)},
$$

for the case when vector $\mathbf{E}$ is parallel to the plane of incidence.

Fig.5 shows simulated dependence of the switch transmittance on the LC refractive index and the incident angle. Liquid crystal layer totally reflects the light when the material's refractive indices and the angle of incidence satisfy the condition of total internal reflection:

$$
n_{L C} \leq n_{\text {glass }} \cdot \sin (\theta)
$$

The figure shows that the total reflection switch design and operating modes are defined by the correlation between the glass prisms material, nematic liquid crystal refractive indices and the angle of incoming beam incidence. Thus to achieve a maximum switching transmittance change it is necessary to make an optimization of these parameters. In such a way it is possible to choose an optimum incident angle for the fixed nematic - glass combination. It should be noted, that the choice of an appropriate incident angle defines the glass prisms shape, which should be simple and suitable for incorporation the switching element into a matrix. 
The thickness of liquid crystal layer does not significantly influence on the switch performance unless it is close to the radiation wavelength value. In this case an evanescent field may reduce the intensity of the reflected beam. This parameter is more important from the driving voltage point of view as long as increasing of the layer thickness leads to the control voltage growth.

In our experiment the switch parameters are justified to operate at $\lambda=633 \mathrm{~nm}$ and to provide the angle of reflection of $45^{0}$. The glass prisms are rectangular shaped to simplify a matrix design.

\section{EXPERIMENTAL RESULTS}

\subsection{Cholesteric mirror switch}

In our experiment the $10 \mu \mathrm{m}$ controllable wave plate (S-cell) is used filled with LC (Merck MLC-9300-100, $\Delta n=$ $0.1154)$ which results a phase difference of $2 \pi+\pi / 2$ and $3 \pi+\pi / 2$ for He-Ne laser radiation $(\lambda=633 \mathrm{~nm})$ by the applied voltages of 1.98 and $2.85 \mathrm{~V}$ correspondingly.

The experimental set-up is shown in Figure 6. The set-up measures the photo detector's responses as functions of applied to the S-cell voltage. The S-cell and the cholesteric mirror are adjusted to operate at $633 \mathrm{~nm}$ wavelength of $\mathrm{He}-\mathrm{Ne}$ laser. The wave plate is driven with a bipolar square wave signal of frequency $\mathrm{f}=1 \mathrm{kHz}$.

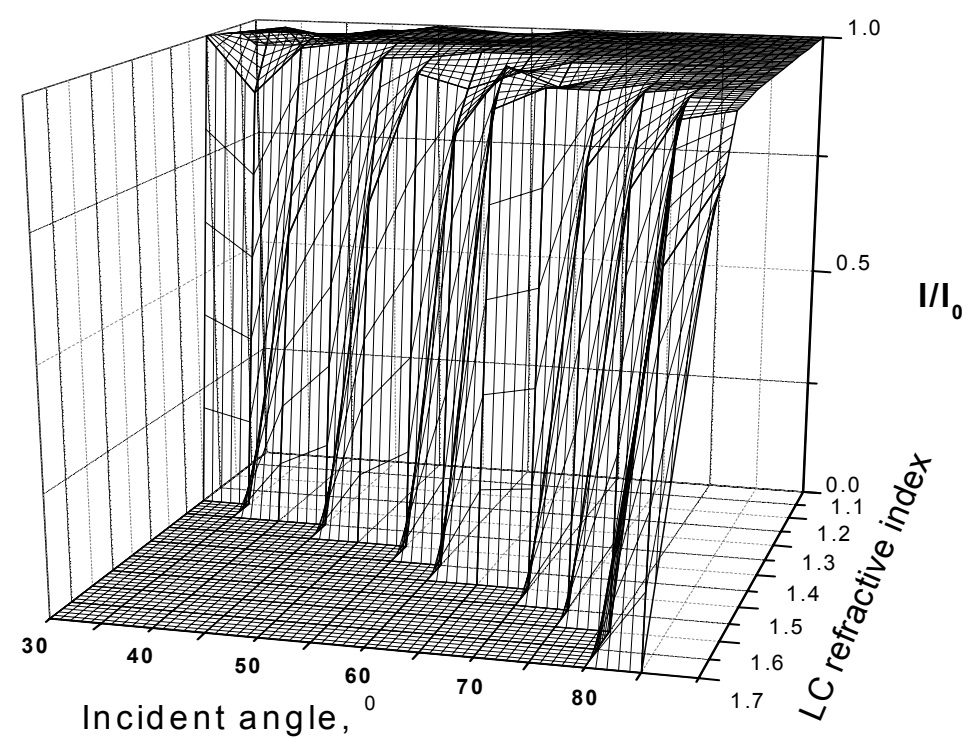

Figure 5.Simulated dependence of the total reflection switch transmittance on liquid crystal refractive index and the incident angle of incoming beam $\left(\mathrm{n}_{\text {glass }}=1.67\right)$. 


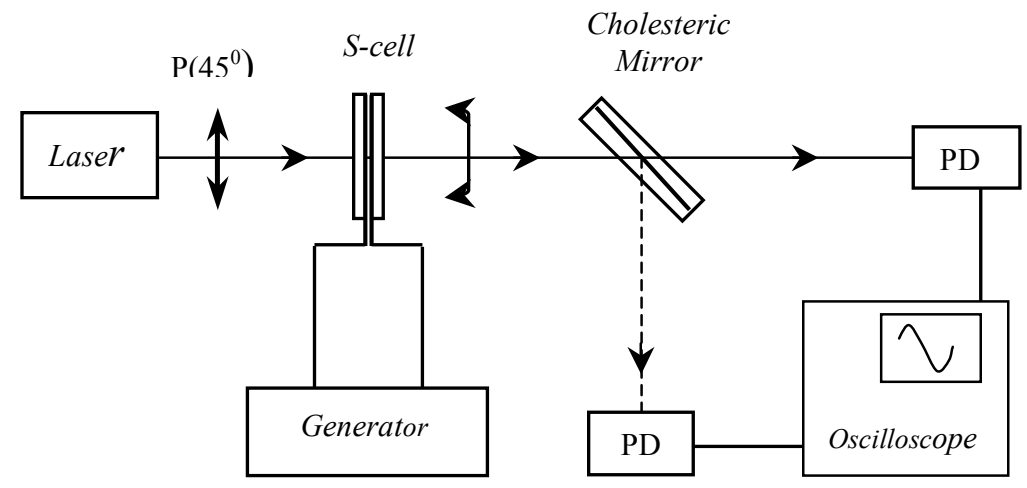

Figure 6. Cholesteric mirror experimental set-up: $\mathrm{P}$ - polarizer, PD - photo-diode.

Cholesteric mirror samples at different LC layer thickness $(5 \ldots 33 \mu \mathrm{m})$ were investigated. The orientation of the cholesteric layers was achieved by rubbing of the glass plates coated by a thin PVA layer.

The material for cholesteric mirror is created on the basis of nematic mixture (Merck MLC-930-100) with an addition of chiral dopant (Figure 7).

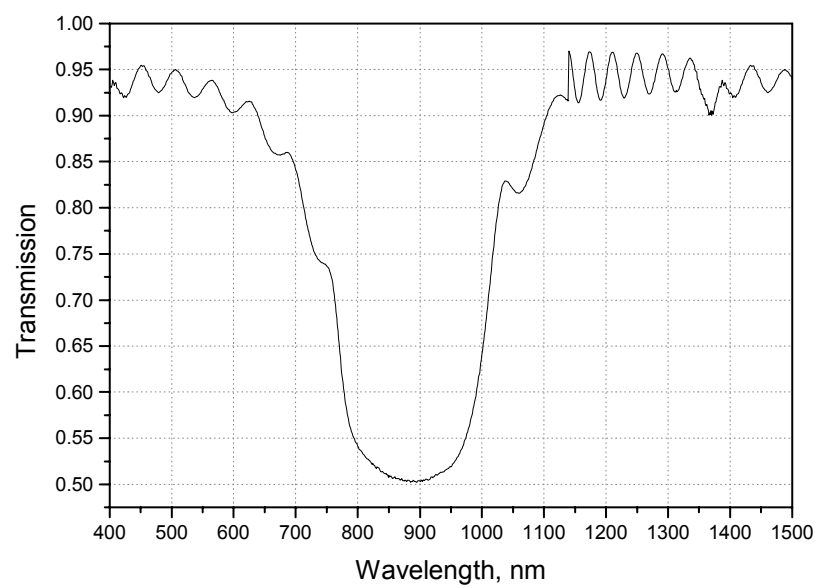

Figure 7. Transmission spectra of the cholesteric mirror sample designed to function at the radiation wavelength of $\lambda=633$ $\mathrm{nm}$ by the angle of $45^{\circ}$.

Figures 8 show the dependencies of electro-optical response on applied to the S-cell voltage for different values of the cholesteric mirror thickness. The voltage from the photo diodes measures the intensity of the transmitted and reflected light. The desired phase differences of $\pi / 2$ and $3 \pi / 2$ were achieved at voltages of 1.98 and 2.85 applied to the controllable wave plate, thus allowing the mirror to function as the core of an optical switch. The switching contrast value is defined by such characteristics of the sample as layer thickness and its alignment quality and also by the incident angle. It is known $[3,4]$, that the maximum reflectivity is increasing with the increase of layer thickness reaching a saturation value at $15 \ldots 20 \mu \mathrm{m}$. On the other hand, layer thickness increase causes a growth of the planar cholesteric structure imperfections that leads to a decrease in reflectivity. Thus, the increase of layer thickness results in the simultaneous decrease of contrast ratio of reflected light and increase of switching contrast ratio for the light transmitted by the mirror (Fig.9). 


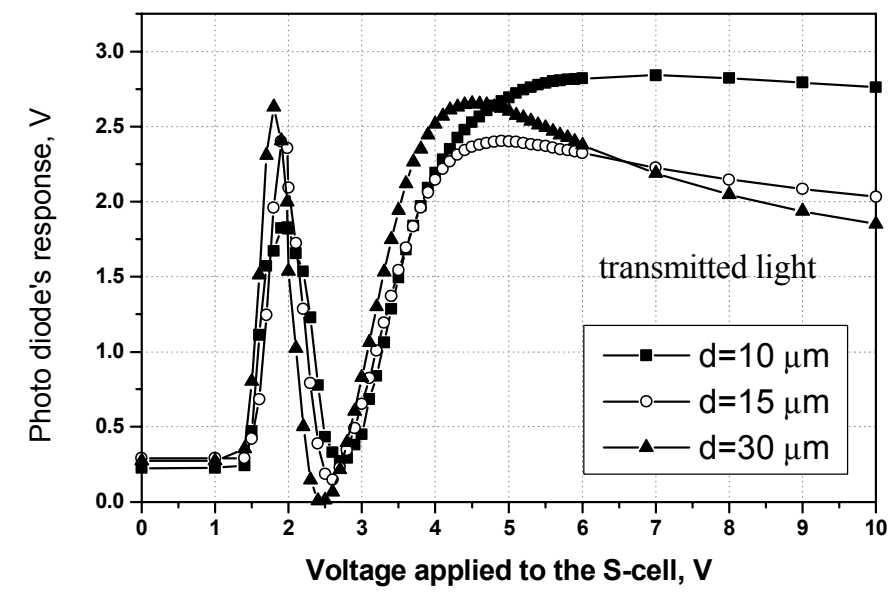

a)

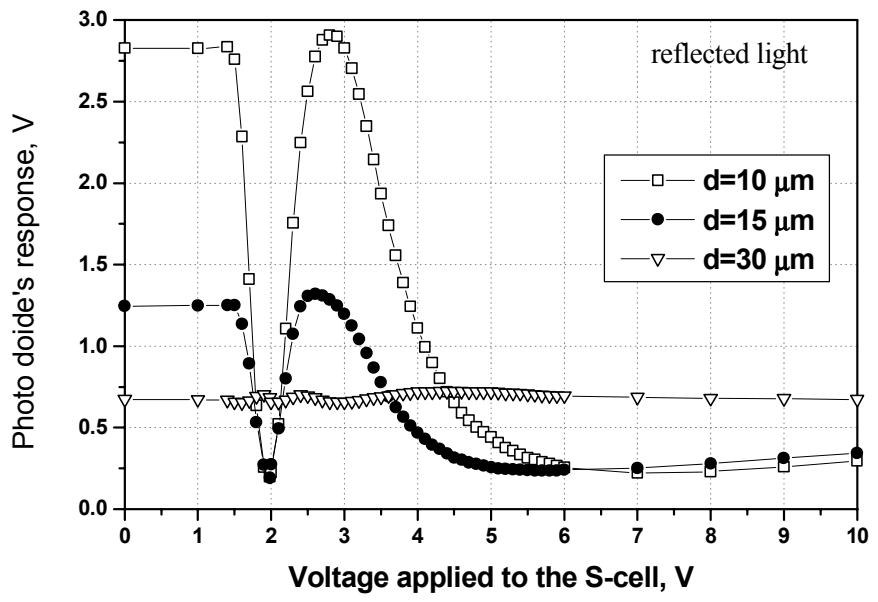

b)

Figure 8. Electro-optic responses of cholesteric mirror switch for various values of the cholesteric layer thickness: a) transmitted light; b) reflected light.

The experimentally found compromise value of layer thickness is of $8 \mu \mathrm{m}$ for $\lambda=633 \mathrm{~nm}$. The equal switching contrast values have been achieved both for the transmitted and reflected light owing to a good cholesteric structure alignment that reduces the reflected beam scattering and loss as a result (Fig.10). The best contrast ratio achieved in cholesteric mirror switch are of 7000:1 (38.5 dB) and of 60:1 (17.8 dB) for transmitted and reflected light correspondingly. The last value can be improved by using anti-reflecting coating.

\subsection{Total reflection switch}

The total reflection switch performance was investigated in the sample on the base of two glass prisms $\left(\mathrm{n}_{\mathrm{g}}=1.6744\right)$ with transparent electrodes. The prisms were coated in a centrifuge with PVA, both substrates were rubbed and then aligned antiparallel to each other. The liquid crystal material (Merck E63, $\mathrm{n}_{\mathrm{e}}=1.7444, \mathrm{n}_{\mathrm{o}}=1.5172, \Delta \mathrm{n}=0.2272$ at $\lambda=$ $589.3 \mathrm{~nm}$ ) was introduced into the cell by capillary action. The thickness of the nematic liquid crystal layer was $25 \mu \mathrm{m}$. 
The experimental set-up is shown in Figure 11. The LC cell (total reflection switch) is designed to operate at $633 \mathrm{~nm}$ wavelength of He-Ne laser. For rectangular prisms used in measurements the incident angle value is $75^{0}$ and the incident light polarization parallel to the direction of the rubbing of the aligning layer.

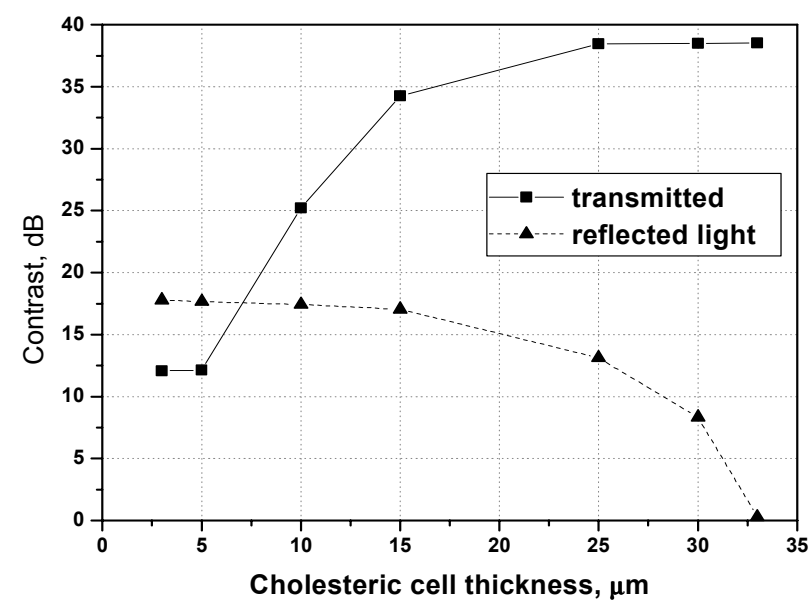

Figure 9.Switching contrast versus the cholesteric cell thickness.

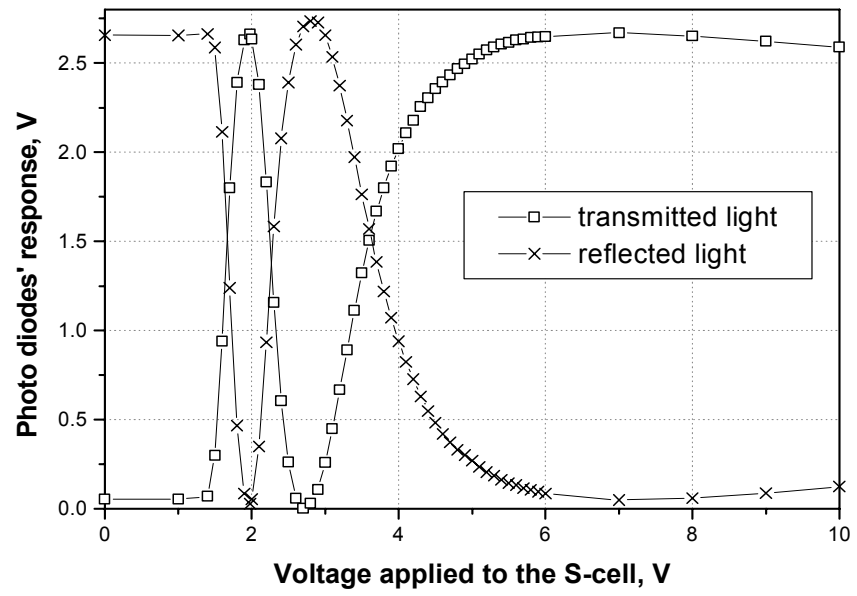

Figure 10. Electro-optic responses for the switch based on the $8 \mu \mathrm{m}$ cholesteric mirror sample.

The total reflection element is driven with a bipolar square wave signal with an amplitude from $0 \mathrm{~V}$ to $35 \mathrm{~V}_{\mathrm{p}-\mathrm{p}}$. The frequency of the driving signal equals to $1 \mathrm{kHz}$.

A typical electro-optic response for the total reflection switch is shown in the Figure 12.

\subsection{Comparison of the switches performance}

We have made simple measurements to compare the mentioned switches performance (see Table I). The glass parts of the both switches did not have an anti-reflection coating on their surfaces. The insertion loss can be reduced by use of a $100 \%$ polarizer, based on cholesteric mirrors. Such a component could have a high transmission, limited only by the optical precision and material orientation defects. 


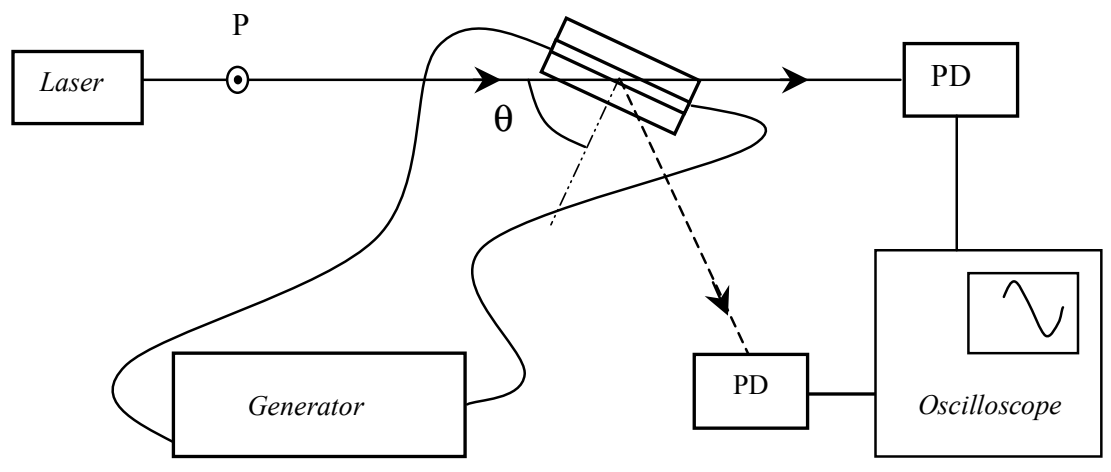

Figure11. Total reflection switch experimental set-up.

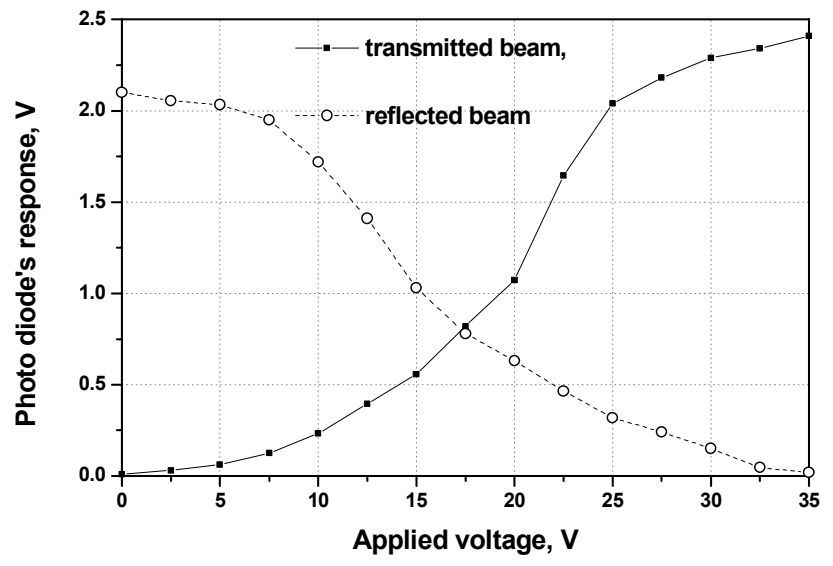

Figure 12.Typical electro-optic response for the total reflection switch.

\section{CONCLUSION}

In conclusion, we have described the operation of two types of all-optical liquid crystal based switches. One of the devices uses the selective reflection effect in cholesteric liquid crystals. We also describe a switching device using the total reflection phenomena on the base of glass prisms and a nematic liquid crystal layer. Prototypes of single switches have been designed and their parameters have been investigated. The first experimental results for a visible radiation indicate that a high switching contrast $(40 \mathrm{~dB})$ and a satisfactory low $(2.5 \ldots 3 \mathrm{~dB})$ insertion loss level can be achieved for the both types of switches. The advantages of the proposed designs are simple and compact structures, electric control, low power consumption and good scalability. Work in progress includes minimizing the insertion loss and cross talk for incorporation into a switch matrix. 
TABLE I The following results were achieved with a helium-neon laser $(\lambda=633 \mathrm{~nm})$

\begin{tabular}{|c|c|c|c|c|c|}
\hline & \multicolumn{2}{|c|}{$\begin{array}{c}\text { Contrast ratio, } \\
\text { DB } \\
\end{array}$} & \multirow[t]{2}{*}{$\begin{array}{l}\text { Insertion loss, } \\
\mathrm{dB}\end{array}$} & \multirow[t]{2}{*}{$\begin{array}{c}\text { Switching time, } \\
\mathrm{ms}\end{array}$} & \multirow[t]{2}{*}{$\begin{array}{c}\text { Drive, } \\
\mathrm{V}\end{array}$} \\
\hline & Transmitted & Reflected & & & \\
\hline $\begin{array}{l}\text { Cholesteric } \\
\text { mirror }\end{array}$ & 38.5 & 17.8 & 2.39 & 280 & 3.55 \\
\hline $\begin{array}{l}\text { Total reflection } \\
\text { switch }\end{array}$ & 42 & 20.5 & 2.89 & 0.34 & $30 p-p$ \\
\hline
\end{tabular}

\section{REFERENCES}

1. C.C. Bowley, P.A. Kossyrev, G.P. Crawford, S.Faris, "Variable-wavelength switchable Bragg gratings formed in polymer-dispersed liquid crystals," App. Phys. Lett. 79, 1 p.6574, 2001.

2. A.Sneh and K. M. Johnson, "High-speed continuously tunable liquid crystal filter for WDM networks," $J$. Lightwave Technol. 14, p.1067, 1996.

3. N. A. Riza and S. Yuan, "Reconfigurable wavelength add-drop filtering based on a banyan network topology and ferroelectric liquid crystal fiber-optic switches," J. Lightwave Technol. 17, p.1575, 1999.

4. J. E. Fouquet, "Compact optical cross-connect switch based on total internal reflection in a fluid-containing planar lightwave circuit," Proc. Optical Fiber Communication Conf., p. 204, OSA, Washington, DC 2000.

5. Qing Li, Xiaole Bai, Jian Zhang and Linsu Tong, "Novel Method for Simulating Optical Properties of Reflective Cholesteric Liquid Crystal Displays," Jpn. J. Appl. Phys. Vol. 40, 11, p.1245, 2001.

6. M. Schubert, "Generalized ellipsometry and complex optical systems," Thin Solid Films, pp.313-314, 1998. 